\title{
Accuracy Of Age Estimation In Kerala Population Using Gustafson's Formula: A Forensic Evaluation
}

\author{
Dr Mini.K.John ${ }^{1}$, Dr Joseph V.J ${ }^{2}$, Dr Arul Dev D.P ${ }^{3}$, Dr Sandeep Lal ${ }^{4}$, \\ Dr Anulekh Babu ${ }^{5}$, Dr Anupama ${ }^{6}$ \\ ${ }^{1,5,6}$ Assistant Professors, Department of Conservative \& Endodontics, Government \\ Dental College, Trivandrum, \\ ${ }^{2}$ Associate Professor, Department of Forensic Medicine, Pushpagiri Medical College, India \\ ${ }^{3}$ P .G student, Department of Oral Surgery, Government Dental College, Calicut \\ ${ }^{4}$ Senior Lecturer, KMCT Dental College, India
}

\begin{abstract}
Gustafson's age estimation includes six age related changes of teeth. He then calculated the regression formula to estimate the age. Six criteria are level of attrition, secondary dentine deposition in pulp, changes in periodontium, root resorption, cementum apposition and root translucency. The aim of the study was to study the physiological changes of teeth according to the Gustafson's criteria and to access the age of the patient according to Gustafson's formula, Maples and Rice and to derive a new formula for age estimation from teeth in Kerala population. The score was calculated from physiological changes in the teeth and a graph was plotted with actual age on one axis and the score calculated on the other. Regression formulae were derived from the obtained graph. 36 extracted anterior teeth were selected. Patient's age and periodontal status was noted at the time of extraction. Teeth were longitudinally trimmed to about 25 micrometer and microscopically evaluated. All parameters of Gustafson's formula were accessed and graded. A regression line was drawn from known age and the total score. A mean error of $4.32 \pm 2.80,3.84 \pm 2.65$ and $4.00 \pm 2.84$ was obtained with Gustafson's formula, Maples formula and newly derived formula respectively. ANOVAs test was done was done to estimate the ages and it is found to be significant.
\end{abstract}

Keywords: Gustafson's formula, Maples formula, newly derived formula, forensic odontology.

\section{Introduction}

Age estimation is a sub discipline of the forensic sciences and should be an integral part of every identification process. Teeth, form a unique part of the human body since it is the most durable and resilient part of human skeleton. Dental evidence can be invaluable in personal identification especially when information relating to the deceased is unavailable. The estimation should be as accurate as possible, since it narrows down the search within the police missing persons files and enables a more efficient and time saving approach [1]. Age estimation is of broader importance in forensic science, not only for the identification purposes of the deceased victims, but also in connection with crimes and accidents.

In forensic odontology, investigations are of three types morphological, radiological and biochemical based on degenerative changes observed on dentition .Gustafson's (1950) age estimation is morphological type. Dentition is not static it is constantly undergoing changes [2]. Appreciable age changes include attrition, periodontal disease, deposition of secondary dentine, root translucency, cementum apposition, root resorption, color changes, increase in root roughness, recession of gingival .These secondary changes in teeth with advancing age are taken into consideration and various studies were done to estimate the age of an individual.

\section{Gustafson's Method Of Age Estimation}

There are many proponents and an opponent for this study. Gustafson's method of age estimation is of great importance in forensic odontology, many formulas and techniques evolved from it. The accuracy of this formula is still disputed; many studies were conducted to prove its authenticity. Gustafson's study was conducted on Europeans in Sweden. It was used on unidentified individuals at Salt River and Tygerberg medico-legal laboratories on a regular basis.Diversity in culture, socioecnomical background, oral hygiene practices and eating habits can alter the wear pattern of teeth. Therefore Gustafson's formula may not be applicable to all individuals in equal magnitude [3].

Age related changes were observed on ground teeth, it includes attrition, periodontitis, secondary dentin deposition, cemental apposition, root resorption and transparency of root. Each sign was ranked from 0 to 3 based on the visual severity of changes and age was estimated. Point values of each age changes are added and an increase in point corresponds to increase in age. A regression line for correlation between age and points was 
drawn. The equation calculated was $\mathrm{y}=11.43+4.56 \mathrm{x}, \mathrm{y}$ is the age and $\mathrm{x}$ is the point value. The error estimated as calculated by Gustafson was $\pm 3.6 y r$.

Many researchers criticized the credibility of Gustafson's formula. Some of them are [4].It can only be used only in dead when tooth is extracted and not in living individuals (Solheim and Annie, 2006)

Assessment of score is subjective (Bang and Ramn 1970)

It is time consuming since too many criteria's need to be evaluated (Maples 1978)

Periodontitis cannot be evaluated due to decomposition of soft tissue in certain cases (Maples 1978)

A common regression line is given for all teeth ignoring eruption time and morphological variations of various teeth (Maples 1978, Solheim 1993)

All criteria's are given equal importance and ignores interrelations between criteria's (Bang and Ramm 1970, Dalitz 1962, Solheim 1993)

Sample size is very less and many of them were from the same patient (Solheim 1993)

Teeth from same individual shows similar wear pattern, statistically it gives a favorable deviation (Maples and Rice 1978, Dalitz (1962)

Dalitz in 1962 re-examined the formula and suggested 5 point score from 0-4, to enhance precision in age estimation. He suggested that root resorption and secondary dentine formation doesn't contribute to the formula and that it could be removed. His study showed a standard deviation of 6yrs

Bang and Ramm used only translucency of dentine as the criteria. Their study proved that age could fairly accurately be determined by these criteria alone. A curvilinear regression was created to express the relation between age and degree of translucency. Johanson (1971) differentiated 7 different stages instead of four. He concluded that root transparency is a criteria that is least influenced by pathological process but Bang and Ramm found the opposite. Reppien (2006) pointed that root transparency increases in diabetics and in case of drug addicts (Maples 1978) [5, 6].

Maple (1978) believes that two criteas, root transparency and secondary dentine formation itself is sufficient enough to give an accurate formula. It is easier to evaluate, observer error may be eliminated .Johanson (1971) excluded attrition, since variation in population was found due to dietary habits and abnormal occlusion Solheim (1993) excluded periodontitis in the criteria and added three more age related changes including surface roughnes,sex and color. He coined two formulas, one including sex and color and other without these criteria's since these factors was not always determinable in all cases.

Gustafson's optimistic standard deviation of $\pm 3.4 \mathrm{yrs}$ has never been confirmed, Johnsons standard deviation of $\pm 5.6 \mathrm{yrs}$ looks too optimistic (Solheim and Anne 2006). Investigations have shown that a standard deviation of $\pm 10 \mathrm{yrs}$ is normal for most methods( Solheim and Annie2006), while others report that these methods have $95 \%$ confidence intervals of approximately \pm 12 yrs (Dalitz 1962). The formulas are more accurate around 40-50yrs and with increasing inaccuracy in younger and especially in older age groups. Another concern is the tendency for overestimation of younger persons and underestimation of older people. (Solheim and Anne 2006) $[6,7]$

\section{Materials And Methods}

The present study was carried out in the Department of Forensic Science, Pushpagiri Medical College, Tiruvalla, Kerala. Total of 36 cases who visited the department of oral and maxillofacial surgery for extraction were taken in the study, 14 cases were excluded out of 50 cases selected since it didn't fit the inclusion criteria's. At the time of extraction age of the patient and extend of periodontal disease was noted. Teeth extracted due to periodontal disease, orthodontic and prosthetic reasons were used in the study. The ethical clearance and consent of the patients were taken prior to the study. . While patients with medical and drug history, trauma from occlusion, abnormal oral habits, congenital anomalies of teeth, pathologies affected teeth, carious, restored teeth, teeth from patients younger than 20yrs were excluded from the study. The design of the study was retrospective cross-sectional. The following dental parameters were studied in each case; attrition, periodontal disease, cementum apposition, secondary dentine deposition, root translucency and root resorption.

The armamentarium used in the study is composed of electric lathe, carborundum stone (rough and smooth), alcohol, xylene, formalin, microscope and slides. Ground section was prepared by hand grinding which was done first with lathe and then with rough carborundum stone until a section of $1 \mathrm{~mm}$ was obtained and at this thickness, the root translucency was noted. Grinding was further done using fine stone until the section of $0.25 \mathrm{~mm}$ thickness is left. Finally, cleaned and dried section was mounted on slide and viewed under microscope for secondary dentine, cementum apposition, and root resorption. The order of preference was incisors-canine-premolars. The scores obtained were tabulated.

Four point score system as per Gustafson's formula [8] (Fig 1) 


\section{Periodontal Disease $(\mathbf{P})$}

$\mathrm{P} 0$ - no periodontitis

P1-beginning of periodontitis

$\mathrm{P} 2$ - periodontal disease more than $1 / 3 \mathrm{rd}$ of the root

P3 - periodontal disease more than $2 / 3$ rd of the root

\section{Secondary Dentin (S)}

S0 - no secondary dentin formation

S1- secondary dentine has begun to form in the upper part of pulp cavity

S2-pulp cavity half filled with secondary dentine

S3-pulp cavity is nearly or wholly filled with secondary dentine

\section{Attrition (A)}

A0 - no attrition

A1 - attrition limited to enamel level

A2 - attrition limited to dentine level

A3 - attrition reaching pulp

\section{Root Transparency $(T)$}

T0-no translucency

T1-beginning of translucency

T2-translucency extending more than $1 / 3^{\text {rd }}$ of root apex

T3-translucency extending more than $2 / 3^{\text {rd }}$ of root apex

\section{Root Resorptio(R)}

R0-no root resorption

$\mathrm{R} 1$-root resorption only at a small isolated spot

R2-resorption limited to cementum

R3-greater area of root affected

\section{Cementum Apposition(C)}

$\mathrm{C} 0$-normal layer of cementum

C1-thickness ofcementum more than normal

$\mathrm{C} 2$-greater layer of cementum

C3-heavy layer of cementum

\section{Results}

The study sample of 36 teeth was divided into four groups according to age( Table 1) with maximum number in 51-60yrs age group( Fig. 1).All six age related changes were evaluated and given scores. Total score was plotted against actual age and a regression line was obtained (Fig. 2) , and a regression formula was obtained- $\mathrm{Y}=4.62 \mathrm{X}+10.57$ ( $\mathrm{X}$ - total score, $\mathrm{Y}$ - Estimated age).With the obtained total score, age estimation was done with Gustafson's formula $\mathrm{Y}=4.56 \mathrm{X}+11.43$ (X-total score, $\mathrm{Y}$ - Estimated age) ,Maples formula $\mathrm{Y}=$ $4.26 \mathrm{X}+13.45$ (X-total score, $\mathrm{Y}$ - Estimated age) and newly derived formula $\mathrm{Y}=4.62 \mathrm{X}+10.57$ (X-total score, Y- Estimated age) (Table 3). In age estimation a mean error of $4.32 \pm 2.80$ yrs with Gustafson's formula, $3.84 \pm$ $2.65 \mathrm{yrs}$ with maples formula and $4.00 \pm 2.84 \mathrm{yr}$ with newly derived formula was obtained. By using Anova test the estimated ages were found to be significant (Tables 4,5$)$

\section{Discussions}

Physiological changes were given score and used to estimate age using linear regression formula and age was estimated with a mean error of \pm 4.00 in contrary to Gustafson's 3.63.In the present study with Maples formula mean error of age is \pm 3.84 while with Gustafson's formula it is $\pm 4.32 \mathrm{yrs}$

The mean error of present study is greater than Singh eta'ls (2004)2.16 yrs, but less than, Bajpai's (2011) 4.86 and Shrigiriwar's (2013) \pm 4.43 yrs. Maples found a mean error of \pm 7 yrs, while in Bajpai's study age estimation with Maples formula, mean error is $\pm 6.43 \mathrm{yrs}^{5}$ and in the current study it is 3.84yrs.Chandler (2013) in his study found a mean error of \pm 11.6 to \pm 13.7 yrs in Western Cape[2,9,10]

In all the researches results show a great fluctuation. The error could be multifactorial including difference in demography, oral habits and hygiene, limited sample size and non specificity of evaluation. Reliable estimation with reproducible result is possible when standardization is strictly followed. A scientific method to accurately measure different criteria's without operator bias should followed. Estimation of age for 
different population shows variant accuracy. Forensic odontologist should develop new technique and validate or challenge existing technology to evolve a more reliable and an authentic method

Conclusion

In the present study it is found that age estimation by Gustafson's formula shows a great variation from the real age when compared to Maples formula and newly derived age estimation formula. Hence applicability of Gustafson's formula in age estimation for Kerala population is not relevant

\section{References}

[1]. Pillai, P.S. and Bhaskar. G. Age Estimation from the Teeth using Gustafson's Method - a Study in India. Journal of Forensic Science1974; 3: 135-41.

[2]. Chandler S. A study to determine the accuracy of Gustafson's method of age estimation on adult teeth when applied to a sample population of Western Cape 2013; www.hdl.handle.net/11394/3264.

[3]. W R Maples M Rice. Some difficulties in Gustafson's dental age estimation. Journal of forensic sciences 1974 .Vol 2; no 1:168-72.

[4]. Amandeep Singh P Gupta. .Physiological changes in teeth as a tool to estimate age. Pacific Journal of Science and technology 2009; 2:956-65.

[5]. Manas Bajpai, Namita Mishra. A comparison of the accuracy of Maples and Rice and newly derived formula for age estimation. A forensic study .Journal of Orofacial Research 2012; 2:1-4.

[6]. Ch Starvianos,D Mastagas,L Stavrianou . Dental age estimation of Adults; A review of Methods and Principles .Research Journal of Medical Sciences 2008; 5: 258-68.

[7]. Metzger, Z., Buchner, A., and Gorsky, M. Gustafson Method for Age Determination from Teeth - A Modification for the Use of Dentists in Identification Teams. Journal of Forensic Science1980. 4:742-9.

[8]. Gustafson, G. Age Determination from Teeth. Journal of American Dental Association 1980; 41: 45-54

[9]. Bang G. and Ramm, E. Determination of Age in Human from Root Dentine Transparency. Acta Octalgia Scandinavic 1970; 28: 335 .

[10]. Burns K R, Maples W R.Estimation of age from individual adult teeth. Journal of Forensic Sciences 1976; $21: 343-56$.

Table1 Percentage distribution of the sample according to actual age

\begin{tabular}{|l|r|r|}
\hline Actual age & \multicolumn{1}{|l|}{ Count } & Percent \\
\hline $31-40$ & 4 & 11.1 \\
\hline $41-50$ & 9 & 25.0 \\
\hline $51-60$ & 12 & 33.3 \\
\hline$>60$ & 11 & 30.6 \\
\hline Total & 36 & 100.0 \\
\hline
\end{tabular}

Fig1. distribution of the sample according to actual age
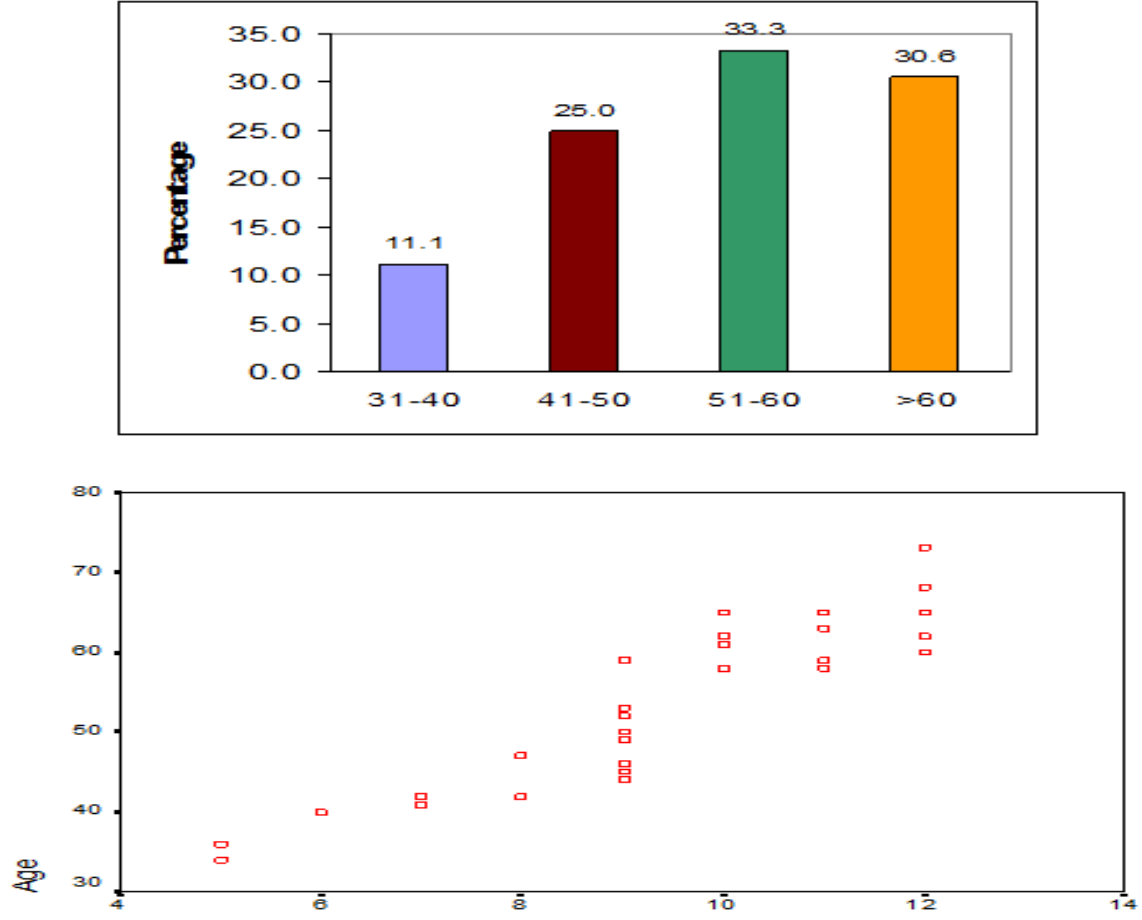

total score

\section{Comparison of estimated age using different formula}

Gustafson formula

$\mathrm{Y}=4.56 \mathrm{X}+11.43(\mathrm{X}$ - total score, $\mathrm{Y}$ - Estimated age) 
Maples formula

$\mathrm{Y}=4.26 \mathrm{X}+13.45$ (X-total score, $\mathrm{Y}$ - Estimated age)

New Formula

$\mathrm{Y}=4.62 \mathrm{X}+10.57(\mathrm{X}$ - total score, $\mathrm{Y}$ - Estimated age $)$

Table2. Comparison of estimated age based on different formula

\begin{tabular}{|l|l|l|l|l|l|}
\hline Formula & Mean & SD & N & F & p \\
\cline { 1 - 3 } Gustafson formula & 54.12 & 8.87 & 36 & \multirow{2}{*}{0.01} & \multirow{2}{*}{0.992} \\
\cline { 1 - 3 } Maples formula & 53.92 & 8.61 & 36 & & \\
\cline { 1 - 3 } New Formula & 53.86 & 9.00 & 36 & & \\
\hline
\end{tabular}

Table 3. Comparison of estimated age based on different formula for different age group

\begin{tabular}{|c|c|c|c|c|c|c|}
\hline Age group & Formula & Mean & SD & $\mathrm{N}$ & F & $p$ \\
\hline \multirow{3}{*}{$31-40$} & Gustafson formula & 36.51 & 2.63 & 4 & \multirow{3}{*}{0.12} & \multirow{3}{*}{0.891} \\
\hline & Maples formula & 36.88 & 2.46 & 4 & & \\
\hline & New Formula & 36.00 & 2.66 & 4 & & \\
\hline \multirow{3}{*}{$41-50$} & Gustafson formula & 49.43 & 3.95 & 9 & \multirow{3}{*}{0.04} & \multirow{3}{*}{0.965} \\
\hline & Maples formula & 48.95 & 3.69 & 9 & & \\
\hline & New Formula & 49.11 & 4.02 & 9 & & \\
\hline \multirow{3}{*}{$51-60$} & Gustafson formula & 56.27 & 4.70 & 12 & \multirow{3}{*}{0.05} & \multirow{3}{*}{0.955} \\
\hline & Maples formula & 55.70 & 4.62 & 12 & & \\
\hline & New Formula & 56.04 & 4.76 & 12 & & \\
\hline \multirow{3}{*}{$>60$} & Gustafson formula & 62.00 & 4.30 & 11 & \multirow{3}{*}{0.03} & \multirow{3}{*}{0.972} \\
\hline & Maples formula & 62.25 & 2.22 & 11 & & \\
\hline & New Formula & 61.86 & 4.39 & 11 & & \\
\hline
\end{tabular}

\section{Comparison of error in estimated age with actual age using different formula}

Table 4. Comparison of error in estimated age with actual age based on different formula

\begin{tabular}{|l|l|l|l|l|l|}
\hline Formula & Mean & SD & N & F & p \\
\cline { 1 - 4 } Gustafson formula & 3.21 & 2.61 & 36 & \multirow{3}{*}{0.36} & \multirow{2}{*}{0.695} \\
\cline { 1 - 3 } Maples formula & 2.77 & 2.30 & 36 & & \\
\cline { 1 - 3 } New Formula & 3.21 & 2.60 & 36 & & \\
\hline
\end{tabular}

Table 5. Comparison of error in estimated age with actual age based on different formula for different age group

\begin{tabular}{|c|c|c|c|c|c|c|}
\hline Age group & Formula & Mean & SD & $\mathrm{N}$ & $\mathrm{F}$ & $\mathrm{p}$ \\
\hline \multirow{3}{*}{$31-40$} & Gustafson formula & 1.11 & 0.64 & 4 & \multirow{3}{*}{0.72} & \multirow{3}{*}{0.512} \\
\hline & Maples formula & 1.00 & 0.20 & 4 & & \\
\hline & New Formula & 1.50 & 0.85 & 4 & & \\
\hline \multirow{3}{*}{$41-50$} & Gustafson formula & 4.32 & 2.80 & 9 & \multirow{3}{*}{0.07} & \multirow{3}{*}{0.932} \\
\hline & Maples formula & 3.84 & 2.65 & 9 & & \\
\hline & New Formula & 4.00 & 2.84 & 9 & & \\
\hline \multirow{3}{*}{$51-60$} & Gustafson formula & 2.02 & 2.24 & 12 & \multirow{3}{*}{0} & \multirow{3}{*}{0.999} \\
\hline & Maples formula & 2.06 & 2.03 & 12 & & \\
\hline & New Formula & 2.04 & 2.27 & 12 & & \\
\hline \multirow{3}{*}{$>60$} & Gustafson formula & 4.35 & 2.43 & 11 & \multirow{3}{*}{0.76} & \multirow{3}{*}{0.478} \\
\hline & Maples formula & 3.32 & 2.26 & 11 & & \\
\hline & New Formula & 4.46 & 2.48 & 11 & & \\
\hline
\end{tabular}

Table 6. Comparison of error in estimated age with actual age based on different age group for different formula

\begin{tabular}{|c|c|c|c|c|c|c|}
\hline Formula & Age group & Mean & SD & $N$ & $\mathrm{~F}$ & $\mathrm{p}$ \\
\hline \multirow{4}{*}{$\begin{array}{l}\text { Gustafson } \\
\text { formula }\end{array}$} & $31-40$ & 1.11 & 0.64 & 4 & \multirow{4}{*}{$3.6^{*}$} & \multirow{4}{*}{0.024} \\
\hline & $41-50$ & 4.32 & 2.80 & 9 & & \\
\hline & $51-60$ & 2.02 & 2.24 & 12 & & \\
\hline & $>60$ & 4.35 & 2.43 & 11 & & \\
\hline \multirow{4}{*}{ Maples formula } & $31-40$ & 1.00 & 0.20 & 4 & \multirow{4}{*}{2.26} & \multirow{4}{*}{0.100} \\
\hline & $41-50$ & 3.84 & 2.65 & 9 & & \\
\hline & $51-60$ & 2.06 & 2.03 & 12 & & \\
\hline & $>60$ & 3.32 & 2.26 & 11 & & \\
\hline \multirow{4}{*}{ New Formula } & $31-40$ & 1.50 & 0.85 & 4 & \multirow{4}{*}{$2.94^{*}$} & \multirow{4}{*}{0.048} \\
\hline & $41-50$ & 4.00 & 2.84 & 9 & & \\
\hline & $51-60$ & 2.04 & 2.27 & 12 & & \\
\hline & $>60$ & 4.46 & 2.48 & 11 & & \\
\hline
\end{tabular}


Figure 1

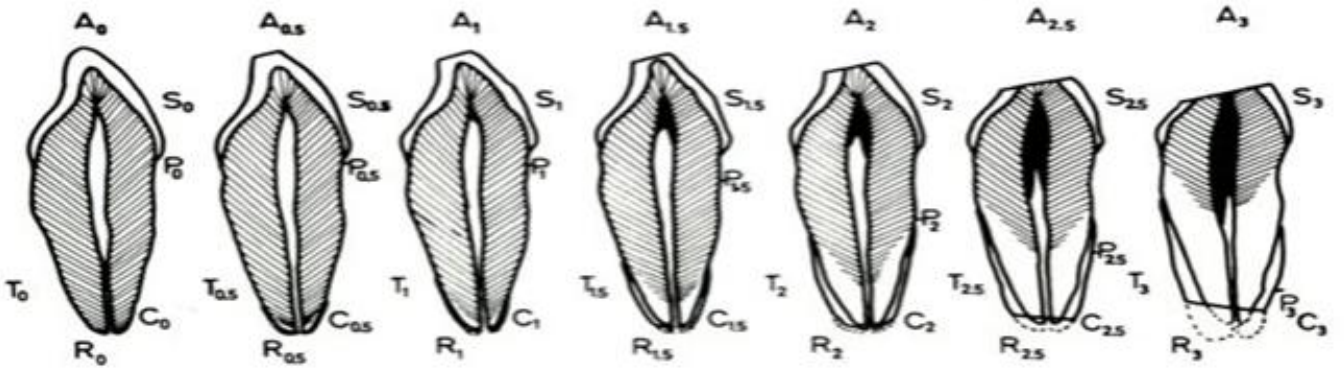

